\title{
The SMRT route to transcriptional repression
}

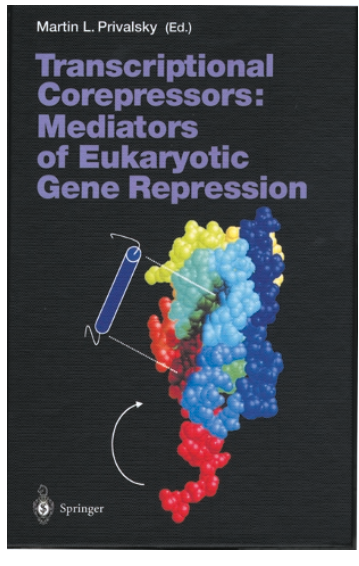

\author{
Transcriptional Corepressors: Mediators of Eukaryotic Gene Repression \\ edited by Martin L. Privalsky
}

Springer-Verlag • December 2000

Hardback $£ 79 / \$ 119$

Jeff Wrana

activity of deacetylases.

This volume, which is part of the Current Topics in Microbiology and Immunology review series from SpringerVerlag, is edited by Martin Privalsky, who brings together a number of reviews on transcriptional repression with the main focus being on pathways that involve histone deacetylases. The reviews attempt to make sense of the dizzying array of proteins and protein complexes that regulate histone deacetylation, and place them in a framework in which to understand their contribution to the overall transcriptional program of a cell. The quality of the production is reasonable but outdated, as all the figures are in black and white. This is disappointing because some of the more complex figures would have been far more approachable with the judicious use of colour. That being said, in large part the volume succeeds in providing well-written overviews that outline the current state of knowledge in the field.

Without a doubt, the authors succeed in conveying to the reader the enormous complexity of histone deacetylation. For instance, the corepressor $\operatorname{Sin} 3 \mathrm{~A}$ is found in a complex with at least 12 other polypeptides, and the NuRD complex is estimated to have a relative molecular mass of about $1^{\wedge}$ million. The task of identifying, dissecting and 'rebuilding' these enzymatic complexes to understand what makes them tick is still in its infancy, and there are many uncertainties in the field. Because of this, there might be a strong tendency in a review to gloss over the complex details of histone deacetylation in order to present a polished story. To their credit, the authors do not shirk from delving into the minutiae of the process, thus making the volume useful to both expert and dilettante alike. Nor do they avoid discussing the unanswered questions and uncertainties that are at issue in this field. This will undoubtedly assist the non-expert to grasp the importance of current contributions to the primary literature.
As a primer for understanding the nuts and bolts of histone deacetylation, this review volume clearly succeeds. Where I have some quibbles is in the amount of overlap between chapters. For instance, the reader is taken through in-depth descriptions of the Sin 3 and NuRD corepressor complexes in several chapters, and the role of corepressors in nuclear hormone receptors is the common theme of two separate chapters. This leads to considerable redundancy and a very real sense of déjà $v u$ while reading different sections of the book. The coverage of transcriptional corepressors in Drosophila development and in the mammalian Smad signalling pathway are thus a welcome relief that start to address the biological role of transcriptional repression. Similarly, the chapter on the retinoblastoma protein provides a clear, if brief glimpse into the role of transcriptional repression in cell growth and differentiation.

But what this review volume has in depth, it lacks in breadth, as coverage of the role that co-repressors play in the Myc-Mad-Max transcription factor network is notably absent. In addition, methylation, insulators and imprinting — which all have important roles in mammalian biology and disease - receive only passing reference. Less overlap between the chapters would have allowed coverage of these areas and expanded the scope of the volume. These additions would have also helped place the role of histone deacetylation in a broader biological context. As a series of reviews that focuses specifically on the field of transcriptional repression via regulated deacetylation of histones, however, this volume hits its mark and will provide an excellent resource for researchers in the field or those contemplating studies in this interesting area.

Jeff Wrana is in the Samuel Lunenfeld

Research Institute, Mount Sinai Hospital, 600 University Avenue, Toronto M5G 1X5,

Canada.

e-mail:wrana@mshri.on.ca 